\title{
Exercise and Sleep in Community-Dwelling Older Adults
}

\author{
Miranda Varrasse ${ }^{1,2}$ • Junxin $\mathrm{Li}^{2,3}$ • Nalaka Gooneratne $e^{3,4}$
}

Published online: 14 October 2015

(C) Springer International Publishing AG 2015

\begin{abstract}
Insomnia and other sleep complaints are highly prevalent in community-dwelling older adults yet often go under detected. Age-related physiological changes may affect sleep, but sleep disturbances and complaints should not be considered normal in this population. Various physiological, psychological, and social consequences have been associated with insomnia and sleep complaints. Treatment options are available so it is imperative to diagnose and treat these individuals to promote healthy aging. Exercise is known to have a wide variety of health benefits, but unfortunately most older adults engage in less exercise with advancing age. This paper describes agerelated changes in sleep, clinical correlates of insomnia, consequences of untreated insomnia, and nonpharmacological treatments for insomnia in older adults, with a focus on the relationship between exercise and sleep in community-dwelling older adults with insomnia or sleep complaints. Possible mechanisms explaining the relationship between exercise and sleep are discussed. While the research to date shows promising evidence for exercise as a safe and effective treatment for insomnia and sleep complaints in community-dwelling older adults, future
\end{abstract}

This article is part of the Topical Collection on Sleep Epidemiology

Miranda Varrasse

mvarr@nursing.upenn.edu

1 NewCourtland Center for Transitions and Health, School of Nursing, University of Pennsylvania, Philadelphia, PA, USA

2 Center for Integrative Science in Aging, School of Nursing, University of Pennsylvania, Philadelphia, PA, USA

3 Center for Sleep and Circadian Neurobiology, School of Medicine, University of Pennsylvania, Philadelphia, PA, USA

4 Division of Geriatric Medicine, School of Medicine, University of Pennsylvania, Philadelphia, PA, USA research is needed before exercise can be a first-line treatment for insomnia and sleep complaints in this population.

Keywords Insomnia $\cdot$ Sleep disturbances $\cdot$ Older adults Exercise

\section{Introduction}

Sleep disturbances are highly prevalent in older adults, with insomnia being the most common sleep disorder [1]. Insomnia is defined as "a chronic or acute sleep disorder characterized by a complaint of difficulty initiating, and/or maintaining sleep, and/or a subjective complaint of poor sleep quality that result in daytime impairment and subjective report of impairment" [2]. Exercise is thought to be a safe, efficacious, and cost-saving intervention to promote health and quality of life [3] and has been used in many studies as an intervention to improve insomnia or sleep complaints in community-dwelling older adults $[4,5 \cdot, 6-8]$ and to improve sleep quality in community-dwelling sedentary older adults $[9,10 \bullet, 11 \bullet]$. This review briefly describes the prevalence of insomnia, agerelated changes in sleep architecture, consequences of untreated insomnia, as well as nonpharmacological treatments for insomnia in older adults and then focuses on synthesizing the association between exercise and insomnia or sleep complaints in community-dwelling older adults by reviewing recent literature (2008-2015) and exploring mechanisms by which exercise can improve sleep.

\section{Prevalence of Insomnia}

The prevalence of insomnia increases with age and varies depending on how it is defined, the rate of incidence, 
remission, and relapse. Epidemiological studies have reported a prevalence of $20-40 \%$ for nighttime insomnia complaints and 10-20\% when daytime symptoms of fatigue or impaired concentration were included in the criteria for insomnia [12]. When more stringent diagnostic nosologies are applied, the prevalence decreased to about 2-5\% [13]. The incidence rate for insomnia symptoms ranged from 5 to $7.97 \%$ per year in two studies of older adults $[14,15]$. About $50 \%$ of people with insomnia symptoms will go into remission in a 1 -year follow-up $[15,16]$. Unfortunately, less than $15 \%$ of patients with insomnia receive treatment or consult a healthcare provider [17].

\section{Age-Related Changes in Sleep}

It is widely known that there are many measurable physiological age-related changes that affect sleep due to changes in sleep homeostasis and the circadian pacemaker, the suprachiasmatic nucleus (SCN), that influences circadian phase [1]. The degeneration of the $\mathrm{SCN}$ results in weaker and more disrupted circadian rhythms. Older adults also go to bed earlier and wake up earlier in relation to their endogenous melatonin secretion. Taken together, these findings suggest that aging is associated not only with phase advance of sleep and circadian rhythms but also with a decreased melatonin output [18].

Elements of sleep architecture change with age. Compared to people with a younger age, older adults are susceptible to decreased total sleep time, early morning awakenings, sleep fragmentation, and less proportion of slow wave sleep (SWS) [1]. In addition to the physiologic age-related changes in sleep architecture, sleep disturbances are much more common among people with poor health.

Another interesting aspect of sleep in older adults is the decoupling of objective changes in sleep and subjective perception of sleep; physiologic changes that occur with aging alone may not be responsible for poor sleep [19•, 20•, 21•]. In a cross-sectional analysis of 2006 the US Behaviors Risk Factor Surveillance System, investigators found that advancing age was not associated with increase in self-reported sleep disturbance, tiredness, or lack of energy, suggesting sleep problems in the elderly population are mediated by factors other than physiologic aging alone [20•]. Although the previous study could be subject to cross-sectional biases, results from a longitudinal study also suggest sleep disturbances in older adults are more dependent on physical, environmental, and comorbid health factors rather than on age-dependent sleep changes [21•]. Stranges and colleagues suggest sleep issues are strongly linked to poorer well-being and quality of life, as well as psychiatric comorbidities [19•]. As coexisting illnesses may cause or be exacerbated by sleep problems, it is evident that sleep disorders are a pressing issue in the older adult. Normal physiological changes of aging may have an impact on sleep quality, but sleep problems should not be referred to as an expected change of aging.

\section{Consequences of Untreated Insomnia}

There are major consequences of untreated insomnia. Chronic or acute insomnia symptoms can lead to fatigue and daytime sleepiness; mood disturbances such as irritability, lethargy, anger, depression, and anxiety; somatic complaints such as joint pain, headaches, and muscle aches; impaired concentration and cognitive performance; decreased perception of quality of life; increased risk of cardiac, metabolic, and immune system problems; increased risk of psychiatric problems; and increased risk of accidents [22, 23]. Evidence from several studies supports an increased risk of depression in older adults with insomnia [24-29], with one study reporting that having insomnia symptoms was associated with a $23 \%$ increased risk of developing depressive symptoms [24]. Insomnia in older adults has been shown to be associated with falls, mood and anxiety disorders, and cognitive impairments [4]. The risk ratio of developing heart disease from insomnia symptoms ranged from 1.47 to 3.90 in one meta-analysis, after controlling for age and cardiovascular risk factors [30]. Recent research also suggests that insomnia symptoms may lead to increase rates of prostate cancer [31]. Sleep quality can impact psychological and social parameters in older adults and inefficient sleep has been shown to predict physical decline in subjects over 75 [32].

Insomnia is associated with high levels of healthcare utilization and increased direct and indirect healthcare costs. Ozminkowski et al. (2007) estimated that over a 6-month period for US adults with insomnia, direct and indirect costs combined were $\$ 1253.00$ higher per person compared to matched controls. The cumulative costs of insomnia in Quebec, Canada, were estimated by Morin and colleagues to exceed six billions dollars annually in a population of around eight million people [33].

\section{Nonpharmacological Treatment}

There are various nonpharmacological treatment options such as improving sleep hygiene, stimulus control therapy, sleep restriction, relaxation training, and cognitive behavioral therapy for insomnia (CBT-I) and other sleep disturbances in older adults [34]. Sleep hygiene and CBT-I are the most commonly used approaches to treat insomnia, where CBT-I is usually initiated if addressing sleep hygiene alone is not effective. Sleep hygiene involves several interventions that promote a routine and stable sleep pattern in a nondisruptive environment. CBT-I consists of six to ten sessions with a therapist 
that focus on maladaptive behaviors and cognitive beliefs that may perpetuate insomnia. There is limited evidence to suggest the effectiveness of sleep hygiene as a monotherapy [35]; however, the efficacy of CBT-I for older adults has been shown in many studies [36-38]. One randomized controlled trial showed improvements in global Pittsburgh Sleep Quality Index (PSQI) scores and PSQI sleep-efficiency sub-score in older adults randomized to a self-help and CBT-I treatment group compared to controls [39]. One meta-analysis reported no significant improvements in objective total sleep time (TST) after CBT-I immediately following treatment or at follow-up but significant improvements in subjective TST, suggesting that CBT-I may be effective at improving subjective sleep misperceptions without changing actual sleep length [40].

\section{Exercise and Sleep}

Participation in moderate exercise is known to reduce premature mortality and to protect against some influences of unhealthy lifestyles [41]. According to the Physical Activity Guidelines Advisor Committee in 2008, only about $10 \%$ of the older adult population over the age of 65 meet the national physical activity guidelines needed to receive health benefits from exercise [11•]. Due to the health benefits associated with exercise, it is important to determine if older adults are able to participate in various physical activities. In this section, we aim to explore recent literature for the potential for exercise to serve as a nonpharmacological treatment for insomnia or sleep complaints in community-dwelling older adults. We will discuss the following: (1) What are plausible mechanisms for which exercise can improve sleep? (2) What types of exercise interventions have been used to improve sleep in older adults, and (3) Does exercise improve symptoms of insomnia and/or sleep quality in older adults?

\section{Mechanisms Explaining Relationship Between Exercise and Sleep}

Various mechanisms describing the relationship between exercise and sleep have been presented in the literature. Thermoregulation is one theory proposed to explain the relationship between exercise and sleep. It is postulated that increasing body temperature can activate heat loss and the associated sleep mechanisms [42]. Sleep onset is triggered by decline in body temperature which is mediated by increased blood flow to skin. Exercising causes an increase in core body temperature which can then facilitate the initiation of sleep due to the activation of heat dissipation mechanisms controlled by the hypothalamus [43]. Glotzbach and Heller argue that insomnia involves impairment in nocturnal temperature downregulation [43]. More experimental studies are needed that explore the interactions among sleep, temperature, and exercise in elderly insomniacs.

Anxiety is thought to be a key player in insomnia. Therefore, another plausible mechanism by which exercise can improve insomnia symptoms is through the anxiolytic effects of exercise [44]. However, in a recent study with adult chronic insomniacs, presleep anxiety decreased after acute aerobic exercise but no correlation was observed between the reduction in anxiety and improvement in sleep [45].

The antidepressant effects of exercise may also play a role in the relationship between exercise and sleep. Multiple studies $[4,45,46]$ have shown positive effects of aerobic exercise on sleep quality and correlations between reduction in depressive symptoms and improvement in sleep quality. A more recent study [47] described depression as a mediator/moderator of sleep improvement in adults and older adults [43]. The role of serotonin in insomnia is still controversial. It is thought that chronic insomnia may be associated with low serotonin levels, and rat studies suggest that exercise promotes feedback regulatory mechanisms that may increase serotonin [43].

A common belief is that sleep loss (accompanying insomnia) may increase vulnerability to disease and that one function of sleep is to promote illness recovery. Furthermore, exercise training has been shown to improve immune function. Therefore, another probable link between exercise and sleep is immunologic alterations [43]. A recent study of healthy older adult men found statistically significant decreases in time awake, rapid eye movement (REM) latency, interleukin-6 (IL-6), tumor necrosis factor- $\alpha(\mathrm{TNF}-\alpha)$, and the ratio of TNF- $\alpha /$ interleukin-10 (IL10) and increases in IL-10 levels and self-reported quality of life following a 6-month moderate aerobic exercise training program [48•]. The data suggest that a 6 -month exercise training program can improve sleep in healthy older adults via modification of cytokine profiles [48 ${ }^{\bullet}$. Further research is warranted to determine if the cytokine profiles change in a similar manner in older adults with sleep disturbances.

Other mechanisms through which exercise may improve sleep are body restoration and energy conservation. Investigators suggest that high catabolic activity during wake (increased during exercise) will be favored by anabolic activity during sleep [42]. Additionally, greater energy expenditure during the day is thought to require greater rest in the evening. Youngstedt and colleagues report that exercise can affect sleep through its circadian shifting effects and increased adenosine [42]. It is widely known that circadian desynchronization can disrupt sleep. Approximately one third of older adults are in a chronic state of malsynchronization, and evidence suggests that exercise can elicit circadian phase shifting effects that are comparable to bright light [49]. Acute exercise can deplete peripheral glycogen stores, and it is claimed that adenosine release is provoked by depletion of central glycogen stores. Adenosine release is associated with sleep, so the thought here is that increasing adenosine release will promote sleep [49]. 
It is clear that various mechanisms describing the relationship between exercise and sleep have been explored. Further research into this relationship and the roles of thermoregulation, anxiolytic and antidepressant, body restoration, levels of serotonins and adenosine, as well as circadian shifting potentials of exercise is critical.

\section{Types of Exercise}

Various studies have utilized subjective and objective measures of sleep to determine efficacy of exercise interventions on community-dwelling older adults with sleep complaints or diagnosed insomnia. Five randomized controlled trials (RCTs) $\left[4,6,8,9,10^{\bullet}\right]$ and three secondary data analyses $[5 \cdot 7,11 \bullet]$ were included in this review (Table 1). Four studies involved moderate intensity aerobic and endurance exercise interventions $[4,5 \bullet, 6,7]$; three studies examined low-impact exercise interventions such as tai chi and yoga that involved mindful meditation and slow relaxing movements $[9,10 \bullet]$; and one study examined leisure activity as exercise [11•].

\section{Association Between Exercise and Sleep}

Both short- and long-term experimental studies utilizing lowimpact meditation and relaxation, aerobic, and leisure exercises have shown positive effects of exercise on subjective sleep quality in mild to moderately sleep-impaired older adults. The bidirectional relationship between sleep and exercise and also the intra-individual variability of sleep within one subject have been explored [37].

Positive outcomes of these studies are decreased stage 1 sleep, increase in stage 2 sleep, less wake after sleep onset (WASO), decreased sleep onset latency (SOL), increase in global PSQI sub-scores, improvement in intra-individual variability (IIV) SOL, and increase in sleep quality ratings, indicating that exercise may be beneficial in improving both objective sleep architecture as well as subjective sleep quality. In addition, there were improvements on subscales of the PSQI, such as sleep duration and sleep efficiency among the studies.

In details, one RCT study with moderate intensity exercise found decreased time spent in stage one sleep, increased time spent in stage two, and less awakenings measured by polysomnography following a 12-month program of moderate-intensity endurance training, compared to a control group who received health education only [6]. Seven of the eight studies found improvements in global PSQI scores and sleep onset latency in the exercise groups $[4,5 \bullet, 6,8,10 \bullet, 50]$, and one study found intra-individual variability improvements in time to fall asleep [7]. Another study reported a withinperson association between exercise and general sleep quality rating (SQR) measured on a Likert scale [11•]. Sleep duration, total sleep time (TST), and sleep efficiency (SE) showed significant improvements following the exercise intervention in four studies $[4,5 \bullet, 8,10 \bullet]$. One study looked into the bidirectional relationship between insomnia and exercise and reported that sleepiness negatively affected exercise duration and that TST moderated the daily relationship between TST and nextday exercise [5•]. Another study reported that age, WASO, and SQR were predictors of physical activity and that the location of the activity did not affect SOL, WASO, or SQR [11•].

One systematic literature review with meta-analysis [50] examining six trials sought to answer a similar question: Does an exercise training program improve sleep quality in middleaged and older adults with sleep problems? There was a significant reduction in medication use and global PSQI scores in the exercise groups, but there were no statistical significant differences in sleep duration, efficiency, sleep disturbance, or daytime functioning [50]. One possible reason for the discrepancy between our results and the meta-analysis is that two studies were primarily comprised of middle-aged subjects, and one study did not enroll based on sleep disturbances. The other three studies in the meta-analysis were included in the present review.

In addition to the aforementioned studies conducted in community-dwelling older adults, Richards and colleagues conducted a series of studies to improve sleep in long-term care residents. Exercise, specifically strength training and walking, in combination with individualized social activities resulted in increased total nocturnal sleep in the long-term care residents $[51,52]$.

\section{Implications and Future Directions}

Exercise is a not only a way to improve sleep but also a potential way to promote healthy aging. The studies evaluated in this paper show promising results for the use of exercise to improve sleep in older adults with insomnia and sleep complaints. Daytime sleepiness in older adults was associated with physical functional impairments and decreased exercise frequency [53]. Excessive daytime sleep and sleepiness are typical sedentary behaviors in older adults and have been linked to multiple adverse health outcomes, including disrupted nocturnal sleep, cardiovascular disease, type 2 diabetes mellitus, obesity, and mental disorders, musculoskeletal diseases, and neurodegenerative diseases [54-56]. Furthermore, sleep fragmentation has been showed to be associated with poorer physical function [57]. Promoting exercise may be an effective approach for older adults to improve sleep continuity, reduce daytime sedentary behaviors, enhance homeostatic and circadian processes of sleep, and improve other aspects of health.

Exercise and sleep can be mutually beneficial. A better night sleep may increase older adults' capacity to exercise, such as increasing motivation, duration, and frequency of exercise, and participating in physical activity may promote a better night sleep. In a large population-based study of older 


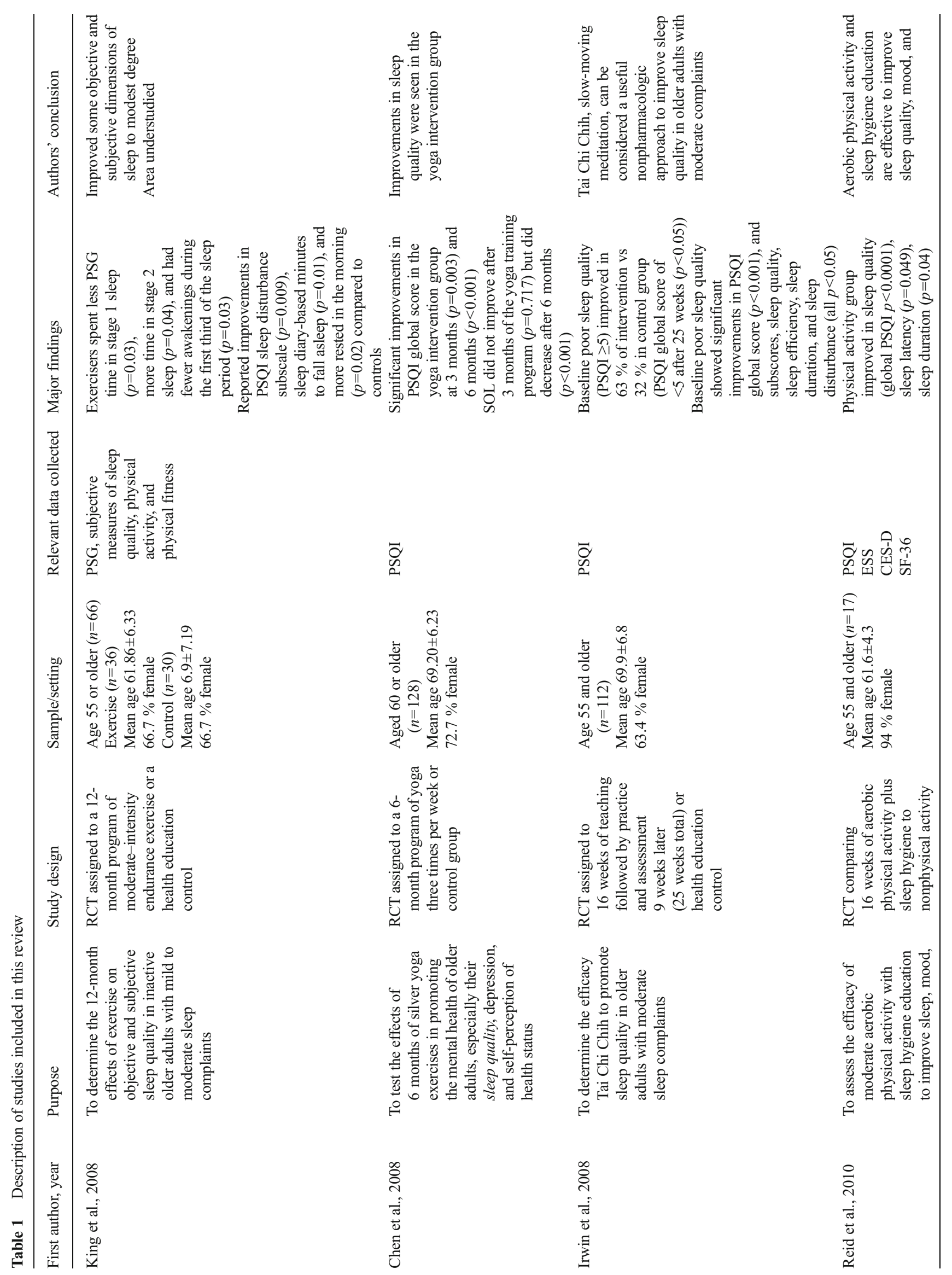




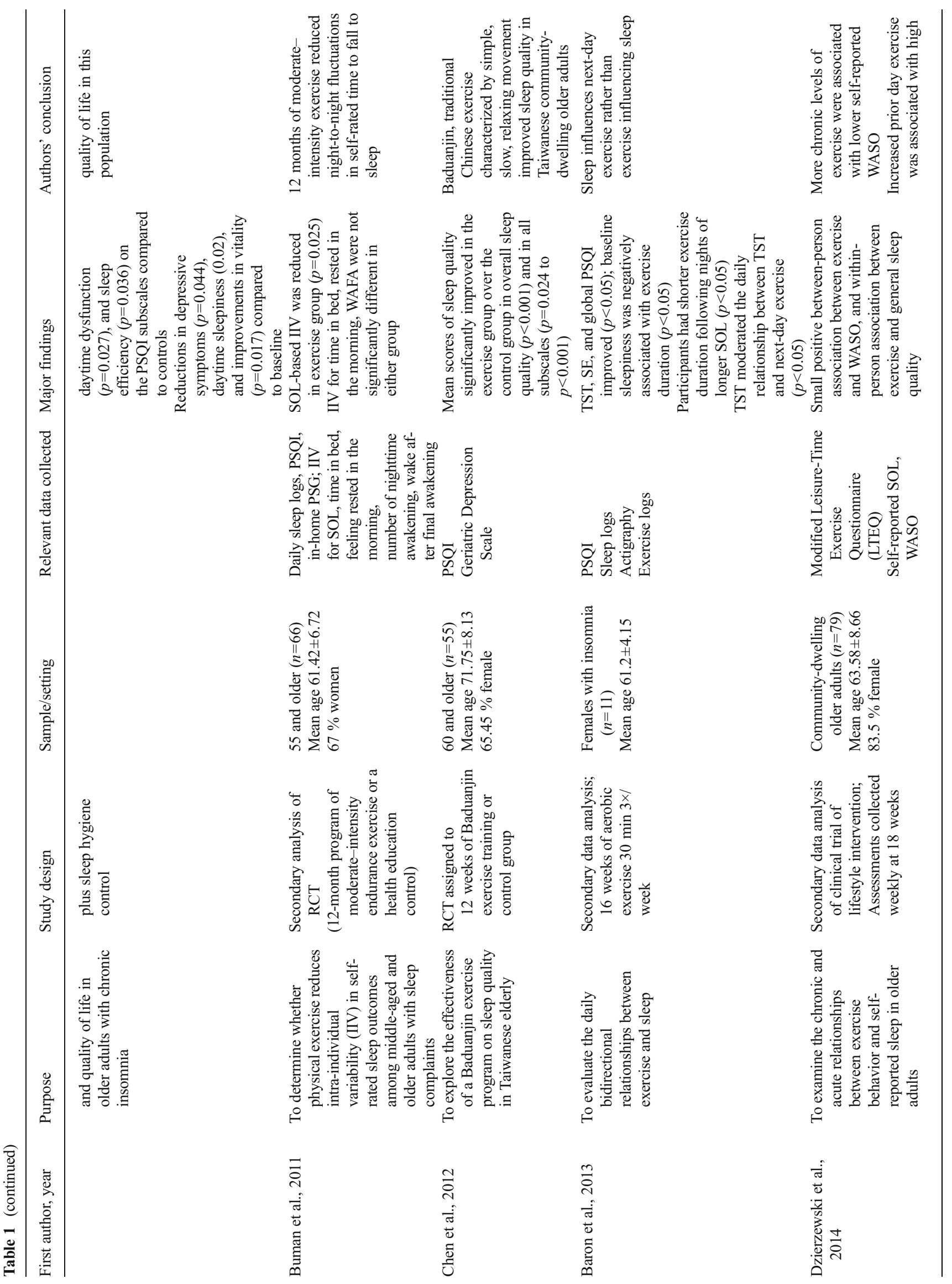


adults in Japan, habitual physical activity was related to both a lower prevalence and incidence of insomnia, especially the symptom of difficulty in maintaining sleep [58 $]$. However, both physical exercise and sleep decline with age. Intervening on either the sleep or exercise component may improve overall health and quality of life in older adults.

Taking into account the results presented on both outcomes of these studies and also possible mechanisms to explain the relationship between sleep and exercise, certain questions arise. First, does the exercise need to be intense aerobic physical activity or is increasing leisure activity enough to improve the sleep quality of older adults in the community? Are there specific exercises that may be safe and effective to improve sleep in older adults with multiple chronic conditions? Could exercise be used as an adjunct to other therapies such as CBT-I? Does the quantity and quality of exercise (aerobic exercise, strength training/endurance, yoga/stretching, etc.), time of day (morning, evening, night), location (light exposure or not) confound, mediate, or moderate the relationship between exercise and sleep in older adults with insomnia? Does exercise decrease daytime sleep and improve nighttime sleep continuity? Does routine exercise lead to sustained benefits? Comparative effectiveness studies examining different types of exercise interventions in older adults with sleep complaints are warranted, as the studies in this review all compared an exercise intervention to a control group. In addition, further research is warranted to explore causal, directional, and mechanistic pathways between exercise and sleep.

\section{Conclusion}

Insomnia and sleep complaints are public health burdens that affect occupation, physical, and social performance. Treating insomnia and sleep complaints in older adults with multiple chronic conditions and various functional status and impairments is complex. Exercise is one potential safe and effective way to manage insomnia symptoms in community-dwelling older adults, but further research is needed. Future research should employ comparative effectiveness techniques to determine what treatment is the most effective, safe, and costeffective.

Acknowledgments Predoctoral fellowship for Miranda Varrasse sponsored by the National Institutes of Health, Ruth L. Kirschstein National Research Service Award (T32NR009356).

\section{Compliance with Ethical Standards}

Conflict of Interest Miranda Varrasse, Junxin Li, and Nalaka Gooneratne declare that they have no conflict of interest. 
Human and Animal Rights and Informed Consent This article does not contain any studies with human or animal subjects performed by any of the authors.

\section{References}

Papers of particular interest, published recently, have been highlighted as:

- Of importance

1. Bloom HG, Ahmed I, Alessi CA, Ancoli-Israel S, Buysse DJ, Kryger MH, et al. Evidence-based recommendations for the assessment and management of sleep disorders in older persons. J Am Geriatr Soc. 2009;57(5):761-89.

2. NIH. National Institutes of Health sleep disorders research plan. 2011.

3. Passos GS, Poyares D, Santana MG, D'Aurea CV, Youngstedt SD, Tufik S, et al. Effects of moderate aerobic exercise training on chronic primary insomnia. Sleep Med. 2011;12(10):1018-27.

4. Reid KJ, Baron KG, Lu B, Naylor E, Wolfe L, Zee PC. Aerobic exercise improves self-reported sleep and quality of life in older adults with insomnia. Sleep Med. 2010;11(9):934-40.

5. Baron KG, Reid KJ, Zee PC. Exercise to improve sleep in insomnia: exploration of the bidirectional effects. J Clin Sleep Med JCSM Off Publ Am Acad Sleep Med. 2013;9(8):819-24. This study suggests a day-to-day relationship between sleep and next-day exercise; sleep influences next-day exercise rather than exercise influencing sleep.

6. King AC, Pruitt LA, Woo S, Castro CM, Ahn DK, Vitiello MV, et al. Effects of moderate-intensity exercise on polysomnographic and subjective sleep quality in older adults with mild to moderate sleep complaints. J Gerontol Ser A Biol Med Sci. 2008;63(9):9971004.

7. Buman MP, Hekler EB, Bliwise DL, King AC. Exercise effects on night-to-night fluctuations in self-rated sleep among older adults with sleep complaints. J Sleep Res. 2011;20(1 Pt 1):28-37.

8. Irwin MR, Olmstead R, Motivala SJ. Improving sleep quality in older adults with moderate sleep complaints: a randomized controlled trial of Tai Chi Chih. Sleep. 2008;31(7):1001-8.

9. Chen KM, Chen MH, Chao HC, Hung HM, Lin HS, Li CH. Sleep quality, depression state, and health status of older adults after silver yoga exercises: cluster randomized trial. Int J Nurs Stud. 2009;46(2):154-63.

10. Chen MC, Liu HE, Huang HY, Chiou AF. The effect of a simple traditional exercise programme (Baduanjin exercise) on sleep quality of older adults: a randomized controlled trial. Int J Nurs Stud. 2012;49(3):265-73. Qigong traditional Chinese exercise (simple, slow, relaxing movements) resulted in significant improvements in sleep quality in this study.

11. Dzierzewski JM, Buman MP, Giacobbi Jr PR, Roberts BL, AikenMorgan AT, Marsiske M, et al. Exercise and sleep in communitydwelling older adults: evidence for a reciprocal relationship. J Sleep Res. 2014;23(1):61-8. This study suggests a reciprocal relationship between within person exercise and sleep quality in older adults.

12. Ohayon MM. Epidemiology of insomnia: what we know and what we still need to learn. Sleep Med Rev. 2002;6(2):97-111.

13. Roth T, Coulouvrat C, Hajak G, Lakoma MD, Sampson NA, Shahly V, et al. Prevalence and perceived health associated with insomnia based on DSM-IV-TR; international statistical classification of diseases and related health problems, tenth revision; and research diagnostic criteria/international classification of sleep disorders, second edition criteria: results from the America insomnia survey. Biol Psychiatry. 2011;69(6):592-600.

14. Foley DJ, Monjan A, Simonsick EM, Wallace RB, Blazer DG. Incidence and remission of insomnia among elderly adults: an epidemiologic study of 6,800 persons over three years. Sleep. 1999;22(2):S366-72.

15. Gureje O, Oladeji BD, Abiona T, Makanjuola V, Esan O. The natural history of insomnia in the Ibadan study of ageing. Sleep. 2011;34(7):965-73

16. Foley DJ, Monjan AA, Izmirlian G, Hays JC, Blazer DG. Incidence and remission of insomnia among elderly adults in a biracial cohort. Sleep. 1999;22(2):S373-8.

17. Morin CM, LeBlanc M, Belanger L, Ivers H, Merette C, Savard J. Prevalence of insomnia and its treatment in Canada. Can $\mathrm{J}$ Psychiatry. 2011;56(9):540-8.

18. Ancoli-Israel S. In: Amlaner CJF, editor. Basics of sleep guide. 2nd ed. West Chester: Sleep Research Society; 2009.

19. Stranges S, Tigbe W, Gomez-Olive FX, Thorogood M, Kandala NB. Sleep problems: an emerging global epidemic? Findings from the INDEPTH WHO-SAGE study among more than 40,000 older adults from 8 countries across Africa and Asia. Sleep. 2012;35(8): 1173-81. This study suggests sleep problems in older adults are strongly linked to poorer well-being, poorer quality of life, and psychiatric comorbidites.

20. Grandner MA, Martin JL, Patel NP, Jackson NJ, Gehrman PR, Pien $\mathrm{G}$, et al. Age and sleep disturbances among American men and women: data from the U.S. behavioral risk factor surveillance system. Sleep. 2012;35(3):395-406. Advancing age was not associated with self-reported sleep disturbance or tiredness in this study, suggesting sleep problems are mediated by factors other than physiological aging.

21. Martin MS, Sforza E, Barthelemy JC, Thomas-Anterion C, Roche F. Sleep perception in non-insomniac healthy elderly: a 3-year longitudinal study. Rejuvenation Res. 2014;17(1):11-8. Results suggest sleep disturbances in the elderly are dependent on physical, environmental, and health factors, rather than age-related sleep changes.

22. Sateia MJ, Doghramji K, Hauri PJ, Morin CM. Evaluation of chronic insomnia. An American Academy of Sleep Medicine review. Sleep. 2000;23(2):243-308.

23. Chesson Jr A, Hartse K, Anderson WM, Davila D, Johnson S, Littner M, et al. Practice parameters for the evaluation of chronic insomnia. An American Academy of Sleep Medicine report. Standards of practice committee of the American Academy of Sleep Medicine. Sleep. 2000;23(2):237-41.

24. Jaussent I, Bouyer J, Ancelin ML, Akbaraly T, Peres K, Ritchie K, et al. Insomnia and daytime sleepiness are risk factors for depressive symptoms in the elderly. Sleep. 2011;34(8):1103-10.

25. Perlis ML, Smith LJ, Lyness JM, Matteson SR, Pigeon WR, Jungquist CR, et al. Insomnia as a risk factor for onset of depression in the elderly. Behav Sleep Med. 2006;4(2):104-13.

26. Cole MG, Dendukuri N. Risk factors for depression among elderly community subjects: a systematic review and meta-analysis. Am J Psychiatry. 2003;160(6):1147-56.

27. Pigeon WR, Hegel M, Unutzer J, Fan MY, Sateia MJ, Lyness JM, et al. Is insomnia a perpetuating factor for late-life depression in the IMPACT cohort? Sleep. 2008;31(4):481-8.

28. Lee E, Cho HJ, Olmstead R, Levin MJ, Oxman MN, Irwin MR. Persistent sleep disturbance: a risk factor for recurrent depression in community-dwelling older adults. Sleep. 2013;36(11):1685-91.

29. Suh S, Kim H, Yang HC, Cho ER, Lee SK, Shin C. Longitudinal course of depression scores with and without insomnia in non- 
depressed individuals: a 6-year follow-up longitudinal study in a Korean cohort. Sleep. 2013;36(3):369-76.

30. Schwartz S, McDowell Anderson W, Cole SR, Cornoni-Huntley J, Hays JC, Blazer D. Insomnia and heart disease: a review of epidemiologic studies. J Psychosom Res. 1999;47(4):313-33.

31. Sigurdardottir LG, Valdimarsdottir UA, Mucci LA, Fall K, Rider JR, Schernhammer E, et al. Sleep disruption among older men and risk of prostate cancer. Cancer Epidemiol Biomarkers Prev. 2013;22(5):872-9.

32. Crawford-Achour E, Dauphinot V, Martin M, Tardy M, Gonthier R, Barthelemy J, et al. Can subjective sleep quality, evaluated at the age of 73 , have an influence on successful aging? The PROOF study. Open J Preventative Med. 2014;4(2):51-6.

33. Severson CA, Tsai WH, Ronksley PE, Pendharkar SR. Identification of insomnia in a sleep center population using electronic health data sources and the insomnia severity index. J Clin Sleep Med JCSM Off Publ Am Acad Sleep Med. 2013;9(7):65560.

34. Schutte-Rodin S, Broch L, Buysse D, Dorsey C, Sateia M. Clinical guideline for the evaluation and management of chronic insomnia in adults. J Clin Sleep Med JCSM Off Publ Am Acad Sleep Med. 2008;4(5):487-504.

35. McCurry SM, Logsdon RG, Teri L, Vitiello MV. Evidence-based psychological treatments for insomnia in older adults. Psychol Aging. 2007;22(1):18-27.

36. Montgomery P, Dennis J. A systematic review of nonpharmacological therapies for sleep problems in later life. Sleep Med Rev. 2004;8(1):47-62.

37. Morin CM, Colecchi C, Stone J, Sood R, Brink D. Behavioral and pharmacological therapies for late-life insomnia: a randomized controlled trial. JAMA. 1999;281(11):991-9.

38. Alessi C, Vitiello MV. Insomnia (primary) in older people. Clin Evid (Online). 2011;2011.

39. Morgan K, Gregory P, Tomeny M, David BM, Gascoigne C. Selfhelp treatment for insomnia symptoms associated with chronic conditions in older adults: a randomized controlled trial. J Am Geriatr Soc. 2012;60(10):1803-10.

40. Okajima I, Komada Y, Inoue Y. A meta-analysis on the treatment effectiveness of cognitive behavioral therapy for primary insomnia. Sleep Biol Rhythm. 2011;9(1):24-34.

41. Blair SN, Kampert JB, Kohl 3rd HW, Barlow CE, Macera CA, Paffenbarger Jr RS, et al. Influences of cardiorespiratory fitness and other precursors on cardiovascular disease and all-cause mortality in men and women. JAMA J Am Med Assoc. 1996;276(3): 205-10.

42. Montgomery P, Dennis J. Physical exercise for sleep problems in adults aged 60+. Cochrane Database Syst Rev. 2002;4:CD003404.

43. Passos GS, Poyares DL, Santana MG, Tufik S, Mello MT. Is exercise an alternative treatment for chronic insomnia? Clinics (Sao Paulo). 2012;67(6):653-60.

44. Youngstedt SD. Effects of exercise on sleep. Clin Sports Med. 2005;24(2):355-65.
45. Passos GS, Poyares D, Santana MG, Garbuio SA, Tufik S, Mello MT. Effect of acute physical exercise on patients with chronic primary insomnia. J Clin Sleep Med JCSM Off Publ Am Acad Sleep Med. 2010;6(3):270-5.

46. Singh NA, Clements KM, Fiatarone MA. A randomized controlled trial of the effect of exercise on sleep. Sleep. 1997;20(2):95-101.

47. Buman MP, Hekler EB, Bliwise DL, King AC. Moderators and mediators of exercise-induced objective sleep improvements in midlife and older adults with sleep complaints. Health Psychol Off J Div Health Psychol Am Psychol Assoc. 2011;30(5):579-87.

48. Santos RV, Viana VA, Boscolo RA, Marques VG, Santana MG, Lira FS, et al. Moderate exercise training modulates cytokine profile and sleep in elderly people. Cytokine. 2012;60(3):731-5. Moderate exercise training was associated with objective improvements in sleep quality, which may be modulated by a reduction in inflammation and changes in cytokine profiles.

49. Youngstedt SD, Kripke DF, Elliott JA, Rex KM. Circadian phaseshifting effects of a laboratory environment: a clinical trial with bright and dim light. J Circadian Rhythms. 2005;3:11.

50. Yang PY, Ho KH, Chen HC, Chien MY. Exercise training improves sleep quality in middle-aged and older adults with sleep problems: a systematic review. J Physiother. 2012;58(3):157-63.

51. Richards KC, Beck C, O'Sullivan PS, Shue VM. Effect of individualized social activity on sleep in nursing home residents with dementia. J Am Geriatr Soc. 2005;53(9):1510-7.

52. Richards KC, Lambert C, Beck CK, Bliwise DL, Evans WJ, Kalra GK, et al. Strength training, walking, and social activity improve sleep in nursing home and assisted living residents: randomized controlled trial. J Am Geriatr Soc. 2011;59(2):214-23.

53. Chasens ER, Sereika SM, Weaver TE, Umlauf MG. Daytime sleepiness, exercise, and physical function in older adults. J Sleep Res. 2007;16(1):60-5.

54. Gennuso KP, Gangnon RE, Matthews CE, Thraen-Borowski KM, Colbert LH. Sedentary behavior, physical activity, and markers of health in older adults. Med Sci Sports Exerc. 2013;45(8):1493-500.

55. Chastin SF, Ferriolli E, Stephens NA, Fearon KC, Greig C. Relationship between sedentary behaviour, physical activity, muscle quality and body composition in healthy older adults. Age Ageing. 2012;41(1):111-4.

56. Martin JL, Ancoli-Israel S. Sleep disturbances in long-term care. Clin Geriatr Med. 2008;24(1):39-50.

57. Dam TT, Ewing S, Ancoli-Israel S, Ensrud K, Redline S, Stone K. Association between sleep and physical function in older men: the osteoporotic fractures in men sleep study. J Am Geriatr Soc. 2008;56(9):1665-73.

58. Inoue S, Yorifuji T, Sugiyama M, Ohta T, Ishikawa-Takata K, Doi H. Does habitual physical activity prevent insomnia? A crosssectional and longitudinal study of elderly Japanese. J Aging Phys Act. 2013;21(2):119-39. Habitual and frequent physical activity was related to lower prevalence and incidence of insomnia, respectively. 